\title{
Experience with Image Compression Chip Design using Unified System Construction Tools*
}

\author{
Pravil Gupta, Chih-Tung Chen, J.C. DeSouza-Batista ${ }^{\dagger}$ and Alice C. Parker \\ Department of Electrical Engineering - Systems \\ University of Southern California \\ EEB 300, MC 2562 \\ Los Angeles, CA 90089-2562, USA
}

\begin{abstract}
This paper describes the use of Unified System Construction tools under development at the University of Southern California. The goal of the project is to automate the construction of heterogeneous, applicationspecific systems. Key elements of the USC system include multiprocessor synthesis, multi-chip datapath synthesis, memory-intensive synthesis, and multi-chip partitioning. The tools were applied to design of an image compression chip set, and results of using these tools are reported on here. Our results are comparable to manual designs reported in the literature.
\end{abstract}

\section{Introduction}

Communications, entertainment, and other electronic systems are in widespread use. These systems are generally multi-chip, heterogeneous, and application-specific. Chip-level synthesis tools are invaluable for the rapid production of such systems, and such tools are becoming available for general use. System-level tools can also be used to significantly increase a designer's ability to meet a schedule along with a set of performance and cost constraints, but only a few of these tools have been available in the past.

The Unified System Construction (USC) project at the University of Southern California involves the production of an integrated set of system-level tools for synthesizing multi-chip, heterogeneous application-specific systems which meet cost, performance and power constraints. This paper presents the use of these system-level tools to perform a multi-chip design exercise, a JPEG image compression chip set. The focus of the USC project is on realtime systems, such as entertainment and communication technologies, but does not exclude other applications requiring specialized system design. A block diagram of the system is shown in Figure 1.

\footnotetext{
*This work was supported by the Advanced Research Projects Agency and monitored by the Federal Bureau of Investigation under Contract No. JFBI90092.

${ }^{\dagger}$ Supported by Conselho Nacional de Desenvolvimento Cientifico e Tecnologico - CNPQ/Brazil.
}

The user of the USC software first selects a style for the system. Styles currently supported include

- heterogeneous multiprocessors consisting of

- processors interconnected with point-to-point connections, operating in a non-pipelined fashion.

- non-pipelined processors in a ring,

- non-pipelined processors connected by a bus,

- pipelined processors with point-to-point connections,

- multiple custom VLSI chips, communicating asynchronously,

- multiple custom VLSI chips, communicating synchronously, with common clock, and

- memory-intensive modules consisting of a custom VLSI chip and a separate memory chip.

Many other styles of systems are currently under development. Once a style is selected, specialized tools are invoked to complete the design process. Ultimately, any custom VLSI chips which must be synthesized are then processed by the ADAM high-level synthesis system, which produces a cell netlist. This netlist is input to the Cascade Design Automation Chipcrafter Silicon Compiler, and a chip layout is produced.

The following sections give an overview of each major style of design, in the order each was applied to the compression example. The remaining sections describe the image compression system to be designed and detail various design activities conducted using the USC tools.

\section{Synthesis of Memory-Intensive Sys- tems}

A subset of the USC tools performs automatic synthesis of memory-intensive application-specific systems, with emphasis on hierarchical storage architecture design. The storage architecture is closely connected to the datapath of the system, and isolating its synthesis from datapath synthesis may not result in an efficient solution. Therefore, the design of the datapath and storage architecture 


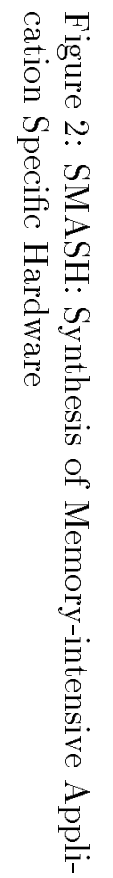



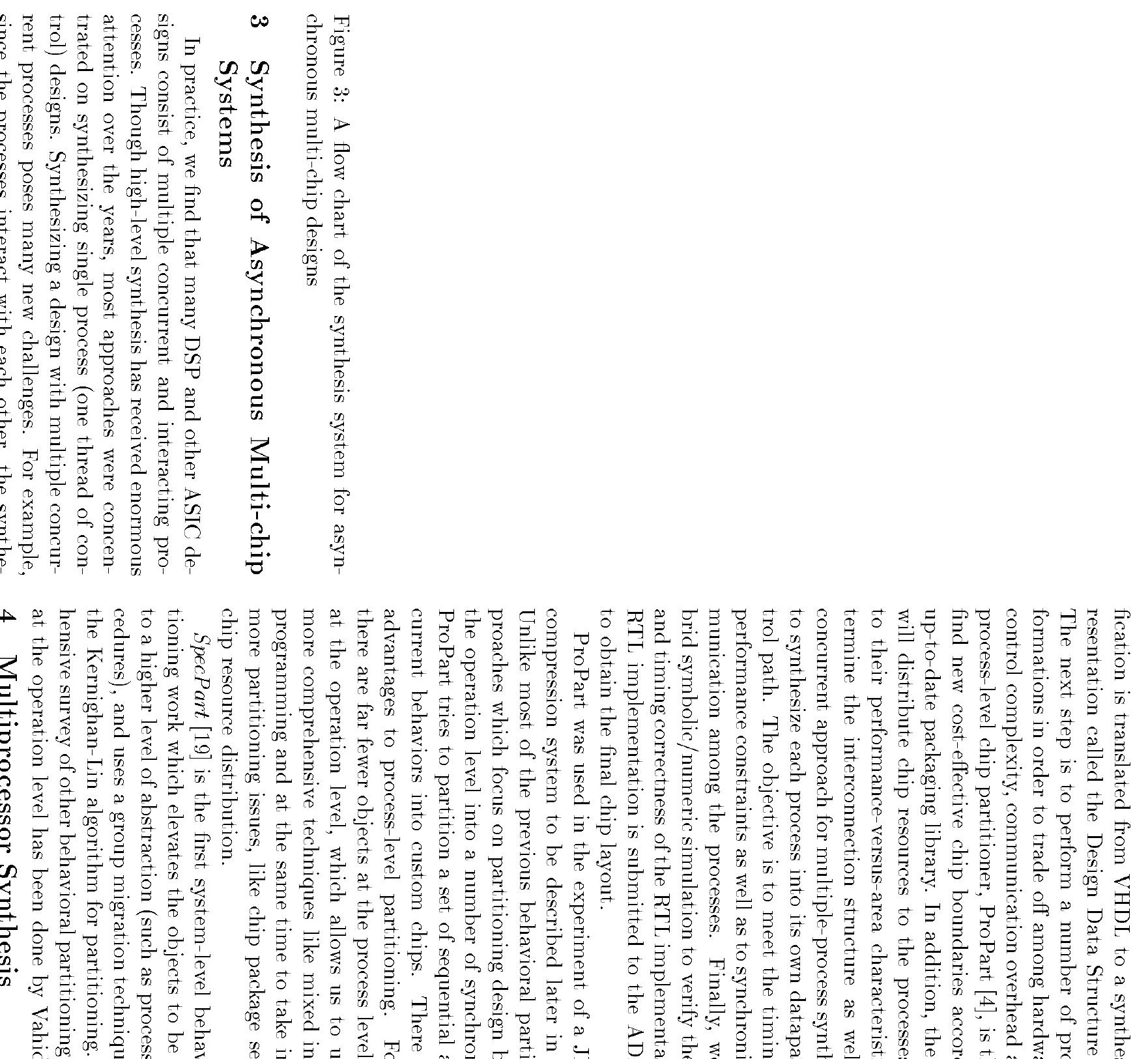



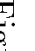




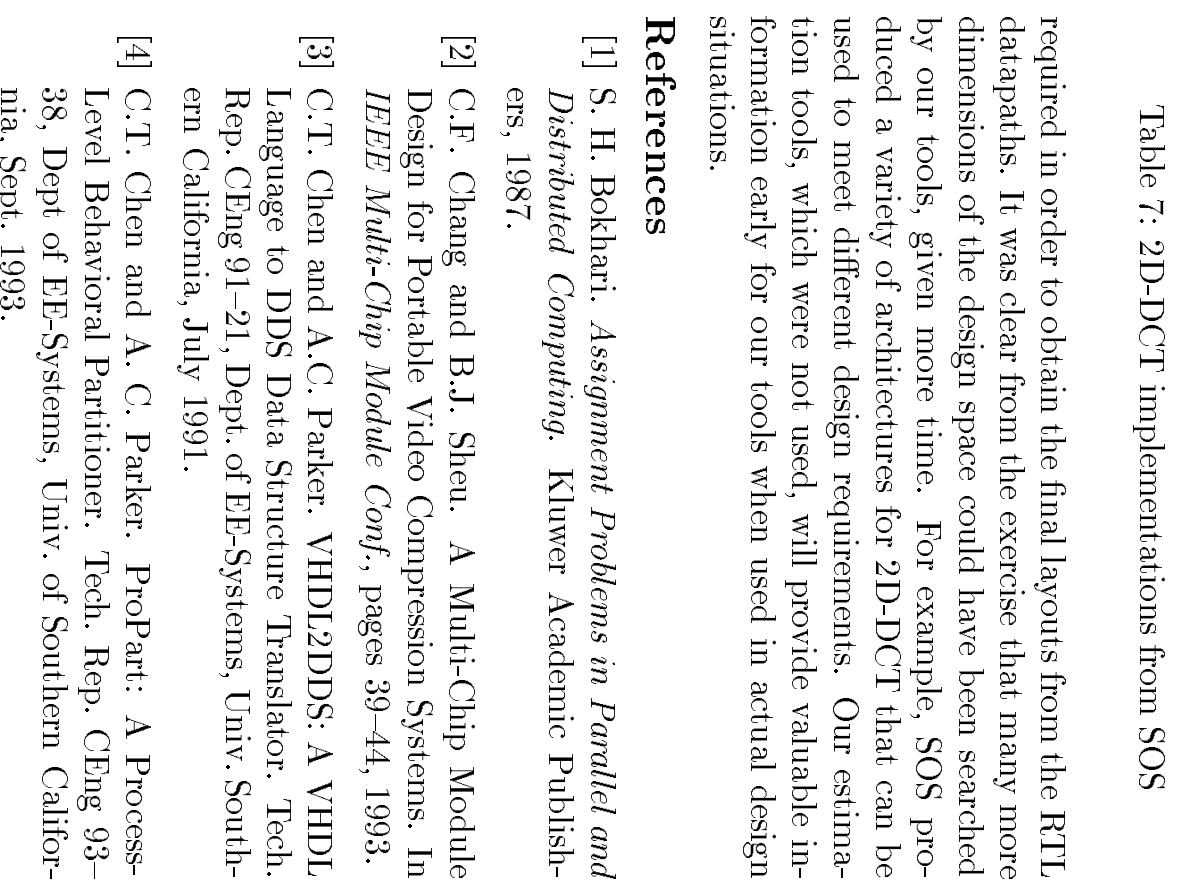

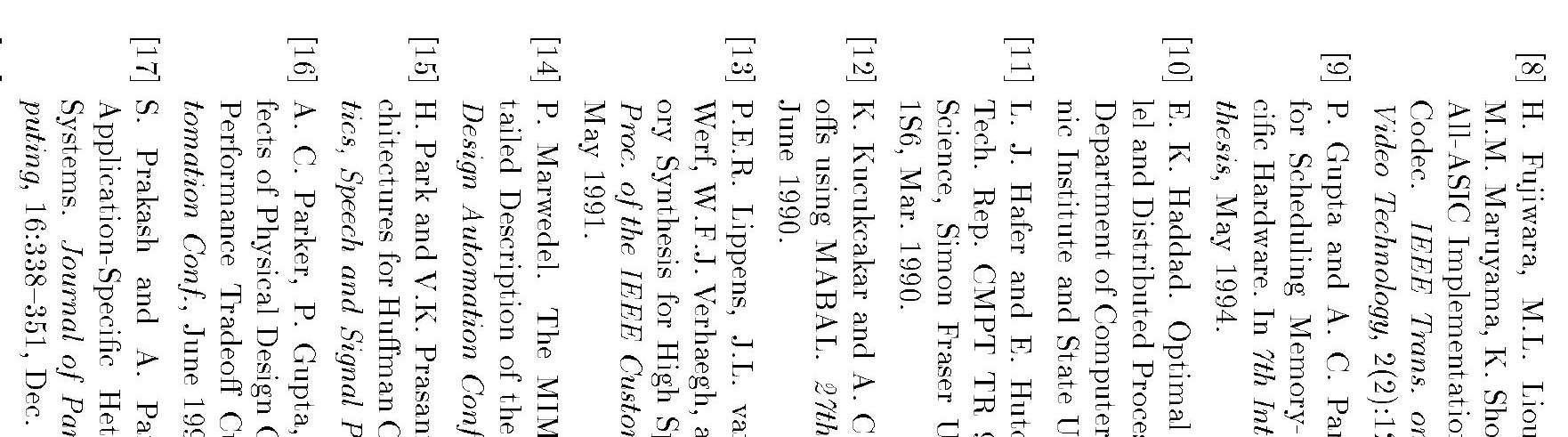

